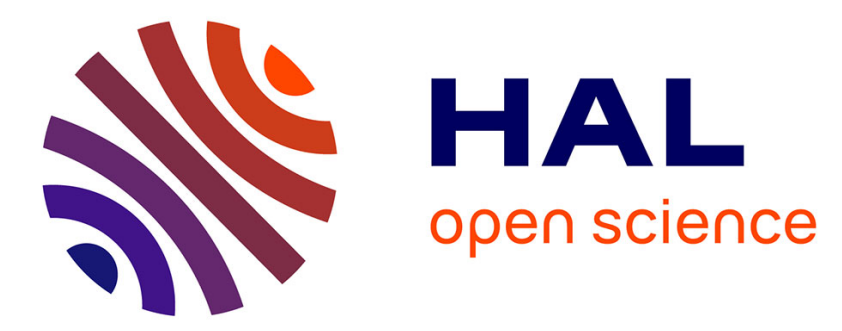

\title{
Optimizing Multi-objective Evolutionary Algorithms to Enable Quality-Aware Software Provisioning
}

Donia El Kateb, François Fouquet, Johann Bourcier, Yves Le Traon

\section{To cite this version:}

Donia El Kateb, François Fouquet, Johann Bourcier, Yves Le Traon. Optimizing Multi-objective Evolutionary Algorithms to Enable Quality-Aware Software Provisioning. The 14th International Conference on Quality Software, Oct 2014, Dallas, United States. pp.85 - 94, 10.1109/QSIC.2014.44 . hal-01090246

\section{HAL Id: hal-01090246 \\ https://hal.inria.fr/hal-01090246}

Submitted on 3 Dec 2014

HAL is a multi-disciplinary open access archive for the deposit and dissemination of scientific research documents, whether they are published or not. The documents may come from teaching and research institutions in France or abroad, or from public or private research centers.
L'archive ouverte pluridisciplinaire HAL, est destinée au dépôt et à la diffusion de documents scientifiques de niveau recherche, publiés ou non, émanant des établissements d'enseignement et de recherche français ou étrangers, des laboratoires publics ou privés. 


\title{
Optimizing Multi-Objective Evolutionary Algorithms to enable Quality-Aware Software Provisioning
}

\author{
Donia El Kateb ${ }^{1,2}$, François Fouquet ${ }^{1}$, Johann Bourcier ${ }^{3}$, Yves Le Traon ${ }^{1,2}$ \\ ${ }^{1}$ Security, Reliability and Trust \\ Interdisciplinary Research Center, SnT, University of Luxembourg, Luxembourg \\ ${ }^{2}$ Laboratory of Advanced Software SYstems (LASSY), University of Luxembourg, Luxembourg \\ ${ }^{3}$ University of Rennes 1, IRISA, INRIA, Rennes, France \\ \{donia.elkateb, francois.fouquet, yves.letraon\}@uni.lu \\ \{johann.bourcier\}@inria.fr
}

\begin{abstract}
Elasticity [19] is a key feature for cloud infrastructures to continuously align allocated computational resources to evolving hosted software needs. This is often achieved by relaxing quality criteria, for instance security or privacy [8] because quality criteria are often conflicting with performance. As an example, software replication could improve scalability and uptime while decreasing privacy by creating more potential leakage points. The conciliation of these conflicting objectives has to be achieved by exhibiting trade-offs. Multi-Objective Evolutionary Algorithms (MOEAs) have shown to be suitable candidates to find these trade-offs and have been even applied for cloud architecture optimizations [21]. Still though, their runtime efficiency limits the widespread adoption of such algorithms in cloud engines, and thus the consideration of quality criteria in clouds. Indeed MOEAs produce many dead-born solutions because of the Darwinian inspired natural selection, which results in a resources wastage. To tackle MOEAs efficiency issues, we apply a process similar to modern biology. We choose specific artificial mutations by anticipating the optimization effect on the solutions instead of relying on the randomness of natural selection. This paper introduces the Sputnik algorithm, which leverages the past history of actions to enhance optimization processes such as cloud elasticity engines. We integrate Sputnik in a cloud elasticity engine, dealing with performance and quality criteria, and demonstrate significant performance improvement, meeting the runtime requirements of cloud optimization.
\end{abstract}

Keywords-MOEAs, Hyper-heuristics, Optimization, Cloud, Software Deployment.

\section{INTRODUCTION}

a) Software Deployment in the Cloud is a MultiObjective Optimization Problem: Cloud Computing paradigm leverages hardware and software resources to offer hosting capabilities that enable customers to get rid of the burden of maintaining their own applications by paying fees that vary on the CPU power consumption per hour or storage usage instead of paying for software license. When hosting a software in the cloud, cloud providers aim at both achieving the quality of service requirements that are stated in the Service Level Agreements (SLA) [33] and at reducing energy consumption. In several cases, these objectives are conflicting: An illustrating example is the one related to Virtual Machines (VMs) allocation: Cloud customers tend to isolate their workloads in separate VMs for security purposes while cloud providers aim at reducing the number of alive VMs to reduce energy consumption in the data-center. The resolution of these objectives has to be performed within acceptable time frame to cope with elasticity features such as resources dynamic autoscaling to meet users needs.

b) MOEAs for Cloud Multi-Objective Optimization Problems Resolution: In [21], the authors have motivated the use of Search Based approaches for the resolution of cloud engineering problems. Multi-Objective Evolutionary Algorithms (MOEAs) [12], [40] is a class of search based approaches that addresses problems in which a decision maker aims at finding a solution that optimizes several conflicting objectives. MOEAs simulate population evolution to produce solutions exhibiting trade-offs between conflicting objectives such as grid jobs scheduler [18] as they are able to automate a set of configurations exploration [10]. A cloud infrastructure, characterized by its dynamic entities, is a typical example of self-adaptive system. MOEAs offer generic and reusable domain exploration capabilities which make them suitable candidate for self-adaptive systems. Self-Adaptive systems require run-time corrective actions [28], that will be made after the identification/selection of the best fitted configuration. A cloud infrastructure can be abstracted by a set of software resources that run on top of Virtual Machines (VMs) dynamically starting/stopping in physical machines. MOEAs are thus used nowadays in several design case studies [18], [15], such as self-adaptive cloud scheduling problems, to maintain conflicting quality characteristics [12], [40] such as system performance, cost and safety. Beyond their applicability for cloud optimizers, MOEAs offer the following advantages to set-up autonomous self-adaptive engines working "at runtime": (i) no need for predefined solutions, (ii) the incremental optimization process can be stopped on-demand, (iii) operating multi-objective optimization and finding trade-offs.

c) MOEAs Tuning is needed to make them appropriate for self-adaptive systems such as a cloud infrastructure: MOEAs are not the standard solution for designing a run-time adaptation engine. A major factor which threatens their adoption for self-adaptive systems such as a cloud infrastructure is the run-time resources consumption, which often requires ad- 
hoc empirical tuning of such algorithms to meet performance needs. Indeed, as in the Darwinian theory [11], [35], the evolution process of MOEAs relies on random mutations to ensure proper domain exploration. This randomness leads to suboptimal performance due to the creation of many dead-born evolution branches. Reed [10] et al report that even if MOEAs usage simplifies the design of automatic configuration engines, empirical fine-tuning of evolutionary search parameters is still needed and can save $70 \%$ of computational costs. These results motivate the need for software engineering techniques to avoid MOEAs ad-hoc tuning and to provide reusable techniques and frameworks for run-time usage.

d) Besides, modern genetics is evolving: Nowadays, modern genetics does not only rely on natural evolution process. Instead, based on the founding work of Muller et al [34], artificial mutation is now widely used to save time and generation cycles for instance to produce genetically modified organisms. Instead of relying only on crossover and natural selection, Muller et al [34] studied artificial mutation using $\mathrm{X}$-Ray to modify a fruit with an anticipated intent. These principles have led the genetic field to build instruments for such selective artificial mutations: the evolution process is accelerated by selecting some specific mutations that contribute to enhance a certain objective.

e) Our proposal to accelerate MOEAs: Going along the same line, we study how such principles could be adapted to MOEAs to accelerate the convergence by guiding the evolutionary algorithms through dynamically selected mutation operators. Our intuition is that operators applied in a smart and artificial way would provide better results than operators applied randomly, and in particular would reduce the number of useless solutions. Thus, the new algorithm we propose is no longer inspired by Darwinian evolution, but by "artificial mutation", based on a smart and dynamic selection of the best mutation operator to apply at a given step. By applying such operators in priority, we aim at orienting the evolution process of a given population in the right direction for the problem to solve.

In this paper, we present an hyper-heuristic [6], called Sputnik, inspired by artificial mutation. Our algorithm takes its name from a virus family which evolves and mutates together with their host in order to perfectly fit their environment and to replicate more quickly. In the same manner, Sputnik algorithm leverages a continuous ranking of operators according to their impact on fitness functions to smartly select dynamically the most relevant mutation operator as the search evolves.

We focus on performance as a key factor for run-time usage to reach faster acceptable trade-offs while saving computation time and generation cycles. For instance, the acceleration Sputnik provides is useful for adaptive systems when a solution/reaction has to be found in a short time. We evaluate our approach on a cloud reasoning engine that is able to continuously provision customers software while handling several conflicting objectives (i.e, isolation, cost). We have integrated Sputnik in the Polymer ${ }^{1}$ framework and evaluated it using

\footnotetext{
${ }^{1}$ http://kevoree.org/polymer/
}

Kevoree $^{2}$ as a models@run.time platform. We have conducted experiments to compare natural selection performance versus Sputnik performance. Our experiments highlight that Sputnik results in a faster convergence by reducing the number of generations while conserving the ability to achieve acceptable trade-offs in our use case. This paper is organized as follows. Section 2 describes the key concepts related to this paper. Section 3 presents Sputnik hyper-heuristic. Section 4 presents validation elements of our approach. Finally, Sections 5 and 6 discuss the related work, our conclusion and future work.

\section{Cloud Optimization, MOEAs Run-time CONSTRAINTS AND HYPER-HEURISTICS}

This section introduces existing approaches that have tackled the cloud multi-objective optimization problem. We also briefly outline MOEAs concepts used in Sputnik and their relevance to drive run-time optimization. In a second step, we give an overview about MOEAs performance issues with a particular focus on run-time usage. Finally, we highlight the role of hyper-heuristics to improve MOEAs algorithms efficiency.

\section{A. Multi-Objective Optimization of Software Deployment in the Cloud}

When moving their applications to the cloud, cloud customers take advantage of an elastic environment in which resources are previsioned/deprovisioned automatically to adapt to variable workload. Cloud elasticity leverages a set of actions that are responsible to move cloud configurations from one state to another. This leads to a very wide set of potential candidate solutions, and consequently to a wide domain to explore in order to find at a given time the best cloud configuration. Different cloud optimization axes have been recently explored over the literature. For instance some of the approaches are cost effective, other approaches are oriented towards performance improvement or security hardening while others are oriented towards achieving an eco-friendly green cloud. In [7], the authors have defined an algorithm called optimal cloud resource provisioning (OCRP) in which they provide an optimal cloud resources provisioning by formulating and solving a stochastic integer programming. The results show that the approach is able to reduce resources costs. In [27], the authors have proposed Mistral, a controller framework that optimizes power consumption, performance, and costs. Mistral is based on a search algorithm that takes into consideration the costs induced by the search algorithm itself. In [9], the authors have built energy profiles to reduce energy consumption in the perspective of designing a green cloud. These energy profiles can be integrated into a monitoring system to reduce energy consumption. In [15], we study how security, isolation requirements and performance objectives can be considered simultaneously in the same optimization process.

\section{B. MOEAs Concepts}

As depicted by [42], genetic based approaches are suitable candidate for scheduling and planning problems. Genetic

\footnotetext{
${ }^{2}$ http://kevoree.org/
} 
Algorithms (GA) are driven by elitism rules that favor the survival of strongest species (best candidate solutions) in analogy to natural selection [40]. They are based on an iterative search process, which involves a set of individuals which are randomly selected and mutated (or mixed using crossover operator) in each iteration to constitute the next generation population. Fitness functions [3] are used to evaluate solutions with regards to a specific optimization problem, in analogy to natural selection where species qualities are evaluated according to their surrounding context. Genetic algorithms introduce changes in the population to create new individuals called offspring through the following operators [39]:

- The crossover operator generates offspring by a genetic recombination of the two selected parents. The resulting offspring maintains some features from each parent, thus maintaining population diversity.

- The mutation operator introduces small changes on an individual with a probability to improve the population diversity [31].

- The selection operator selects a fixed number of fittest offspring for the next generation to maintain a fixed population size and puts good offspring into the next generation with a high probability.

More formally, a multi-objective optimization aims at minimizing a vector function $F(x)=\left(f_{1}(x), f_{2}(x), \ldots, f_{n}(x)\right)$ where $x \in \mathfrak{R}_{n}$ and $F$ is a vector of $n$ objective functions. MultiObjective optimization introduces two important concepts: The Pareto Dominance and the Pareto Optimality [12]: given two solution $X_{1}$ and $X_{2}, X_{1}$ is said to dominate $X_{2},\left(X_{1} \succeq X_{2}\right)$, if $f_{i}\left(X_{1}\right) \preceq f_{i}\left(X_{2}\right)$, for all $i=1, \ldots, n$ and $f_{i}\left(X_{1}\right) \prec f_{i}\left(X_{2}\right)$ for at least one objective function $f_{i}(X)$. A Pareto-optimal front is a curve that groups all solutions belonging to the Pareto-optimal set.

Multi-Objective Evolutionary Algorithms (MOEAs) have been successfully applied in many domains such as finance, logistics and recently in cloud engineering problems. In [10], the authors have provided a taxonomy of the different application domains of MOEAs. Some of these applications have to be used in a run-time context where dynamic parameters adjustment is required such as running vehicles guidance. Runtime optimization has thus to be performed with constrained resources to cope with run-time constraints, the next section highlights these constraints.

\section{Performance of MOEAs at run-time}

The usage of MOEAs in run-time optimization problems [25], for instance load balancing problems which can be seen as a subset of scheduling problems, motivates the need to improve their performance. Several studies have explored the computational costs of MOEAs [29], [40] by evaluating their performance on different problems using various MOEAs categories. According to [26], MOEAs computational costs can be reduced by reducing its algorithmic complexity or the computational costs of the fitness function. Several studies have been focusing on highlighting the computational costs of fitness evaluations while proposing models to reduce its cost. In [5], the authors have tested complex fitness functions, and have proposed the concept of surrogate models to reduce their computational cost. Their approach is based on a gaussian optimization model that evaluates previous fitness functions to estimate future fitness functions scores instead of evaluating real fitness functions. In [38], authors highlight the impact of large populations on the computation time of the Pareto front [24], [23]. In [29], [13], authors conduct experiments and highlight the efficiency loss and overhead introduced by a number of objectives above 3 .

All of these studies highlight MOEAs performance drawbacks and propose specific solutions to improve MOEAs. In this paper, we focus on the notion of hyper-heuristic (described in the next section) to propose a solution with an impact on the algorithm complexity.

\section{Hyper-heuristics: Classification and Objectives}

In search based engineering, hyper-heuristics [6] define methods that act on adapting search parameters over the search process. Hyper-heuristics introduce modifications on the algorithm itself, in order to improve its computational efficiency or effectiveness to handle a specific purpose [36]. Hyper-heuristics rely on machine learning mechanisms [6], to leverage knowledge assessed during each search iteration. For example, in [1] such learning methods store neighborhood information through a neural network to improve a genetic algorithm accuracy. In [22], a learning method is used to prioritize some objectives along the search process.

Hyper-heuristics can be classified according to the following taxonomy [6] depending on the nature of the heuristic used (based on selection or generation methodologies). For each nature, we thus differentiate : (i) Online learning hyper-heuristics which learn while the search algorithm is running, (ii) Offline learning hyper-heuristics which learn from the system before the execution of the search process. The contribution of this paper, falls into the category of hyper-heuristics that embed online learning mechanisms which are based on both selection and generation methodologies to achieve performance improvement over a set of software engineering problems [20], [32]. Indeed, our approach relies on mutation operator prioritization (selection nature) based on the evaluation of past execution efficiency (generation nature).

\section{Sputnik: AN HYPER-HEURISTIC FOR AN EFFICIENT SELF-ADAPTIVE SYSTEMS OPTIMIZATION}

The randomness introduced by natural selection of evolutionary approaches leads to suboptimal performance in terms of computational power and memory usage. Random selection of mutation operators produces useless candidate solutions that lead to computational resources wastage and therefore does not meet run-time optimization constraints. In modern biological studies, after identifying a gene impact on an individual phenotype trait, scientists like Muller et al [34] leverage artificial mutation to directly produce an individual combining the foreseen modification.

Our hypothesis is that the artificial mutation concept can be introduced in evolutionary algorithms to mimic modern biological genetics, thus reducing the number of required generations 
Fig. 1: Sputnik Workflow

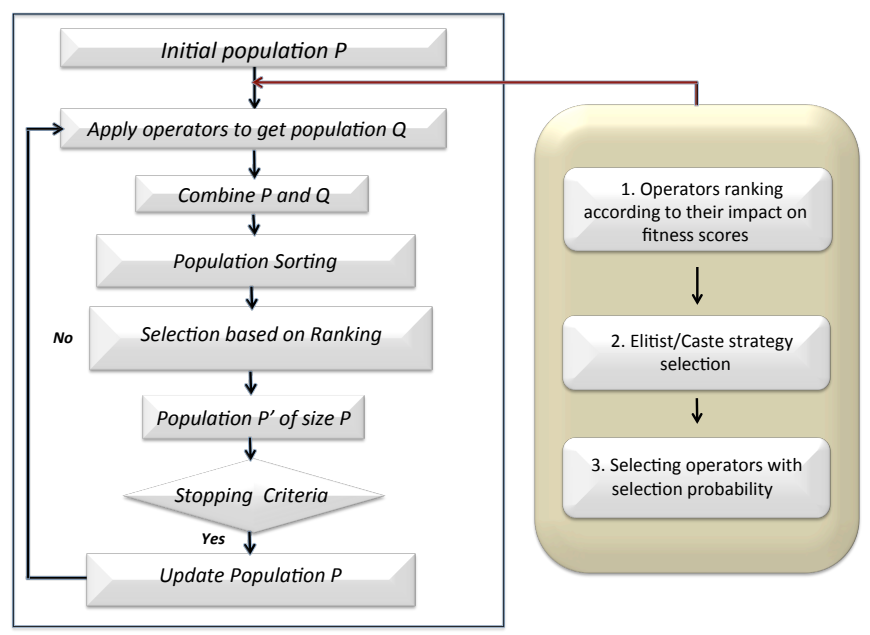

to reach acceptable solutions. The a priori scientific knowledge of a gene modification impact, could be replaced by a continuous ranking and learning approach leveraging execution history. Therefore, in this paper we aim at optimizing MOEAs, by dynamically reducing the usage of mutation operators that are less effective in improving fitness functions scores. At the same time, we maintain the equity of natural selection, to ensure that the modified evolution algorithm is able to reach any solution. Thus, we replace the random mutation operator selection by an hyper-heuristic that detects for each individual the most pertinent operator to apply in order to achieve a faster trade-off.

After each application, mutation operators are classified according to the delta variance they introduce on each fitness function. Internally, Sputnik maintains an elitist group of mutation operators that are relevant to improve a fitness function score. To enhance operators selection, Sputnik, considers the current fitness scores reached by a solution, and selects the most relevant mutator in elite groups to improve next generation ${ }^{3}$. As illustrated in Figure 1, Sputnik takes as inputs an initial population and a generation number. As most of MOEAs variants like (NSGA-II, $\varepsilon$-MOEA, SPEA 2 [40]), the algorithm is based on an individual ranking step according to fitness function evaluation and a non dominating population construction. Sputnik introduces a favoritism operator approach in the mutation process described as follows:

We consider a multi-objective evolutionary optimization of $f$ with $n$ objectives $\left(f_{1}, f_{2}, \ldots, f_{n}\right)$. The average fitness score for a generation $g_{l}$ is defined by $\sum_{i=1}^{n} f_{i} / n$ for each individual in the generation. We define $\triangle_{\text {impact }} f_{g_{l}, o p}$ as the fitness score variation between the average of fitness function evaluation for a generation $g_{l-1}$ and a generation $g_{l}$ that is achieved by the operator $o p$ :

\footnotetext{
${ }^{3}$ http://www.genetics.org/content/111/1/147.short
}

$$
\triangle_{\text {impact }} f_{g_{l}, o p}=\left(\sum_{i=1}^{n} f_{i} / n\right)_{g_{l}, o p}-\left(\sum_{i=1}^{n} f_{i} / n\right)_{g_{l-1}, o p}
$$

Sputnik records the selection occurrence for the different mutation operators that have been involved over the search process. Once all mutation operators have been selected at least once, $\triangle_{\text {impact }} f_{g_{1}, o p}$ is evaluated for all the operators and Sputnik is configured to select the operators that have $\triangle_{\text {impact }} f_{g_{l}, \text { op }}>0$ in the generation $g_{l+1}$ with the Elitist or the Caste strategies. More formally, Sputnik selection function selection $_{o p}$ is specified as follows:

$$
\begin{aligned}
& \text { selection }_{o p} \text { : operators } \times \text { generation } \times \text { objectives } \quad \longrightarrow \quad \text { operator } \times \text { probability } \\
& \left(o p_{1}, o p_{2}, \ldots, o p_{m}\right), g_{l},\left(f_{1}, f_{2}, \ldots, f_{n}\right) \quad \longmapsto \quad o p_{\max \left(\triangle_{\text {impact }} f_{g_{l}, o p}\right)} \times P_{\text {selection }}
\end{aligned}
$$

$P_{\text {selection }}$ depends on Sputnik strategy and is evaluated as follows:

- Elitist Strategy: The operator that has the highest $\triangle_{\text {impact }} f_{g_{l}, o p}$ is selected in the generation $g_{l+1}$ with a high $P_{\text {selection. }}$. This configuration accords higher chance to the "winner operator" to be selected in the next generation. All others operators are selected with a probability $1-P_{\text {selection }}$.

- Caste Strategy: A selection probability is partitioned between operators which have $\triangle_{\text {impact }} f_{g_{l}, o p} \preceq 0$ and is defined as follows:

$$
P_{\text {selection }}=\triangle_{\text {impact }} f_{g_{l}, o p} / \sum_{\text {op } \in \text { operators }} \triangle_{\text {impact }} f_{g_{l}, o p} \text {. }
$$

This configuration gives more equity in terms of selection probability for all operators which have a positive impact on a fitness score.

Sputnik-based mutation operators selection is described in Algorithm 1. In both settings, we set a selection probability of $10 \%$ for pure random selection of operators to not discriminate worst ranked operators. The random selection of operators mimics the natural evolution and aims at giving equitable chances to all solutions, and to any potential mutation operator. This random operators selection ensures a proper exploration of the domain and prevents the solutions to fall into a local minimum. Sputnik keeps $10 \%$ of mutation to give chance to less selected operators to be reintroduced in the elite group of a fitness function. Through this mechanism, we keep a minimal equity of species while conserving $90 \%$ of the Pareto for most efficient mutation.

\section{VALIDATION}

To evaluate Sputnik, we have considered an experimental scenario in which a cloud provider aims at placing several software components related to many customers in the different Virtual Machines running on the top of physical machines. We define a cloud configuration as an architecture model which leverages virtual machines and components (i.e, provisioned software in our context) concepts. Based on our architectural model, an Individual represents a solution vector $X$ that corresponds to a cloud infrastructure model. The reader may refer to our open source Polymer framework which gives ample details about our architectural model ${ }^{4}$. A gene corresponds to a component, a virtual machine or a physical machine in our model. A population corresponds to a set of cloud infrastructure models. A genetic mutation operator corresponds to an elementary

\footnotetext{
${ }^{4} \mathrm{http}: / /$ kevoree.org/polymer/
} 


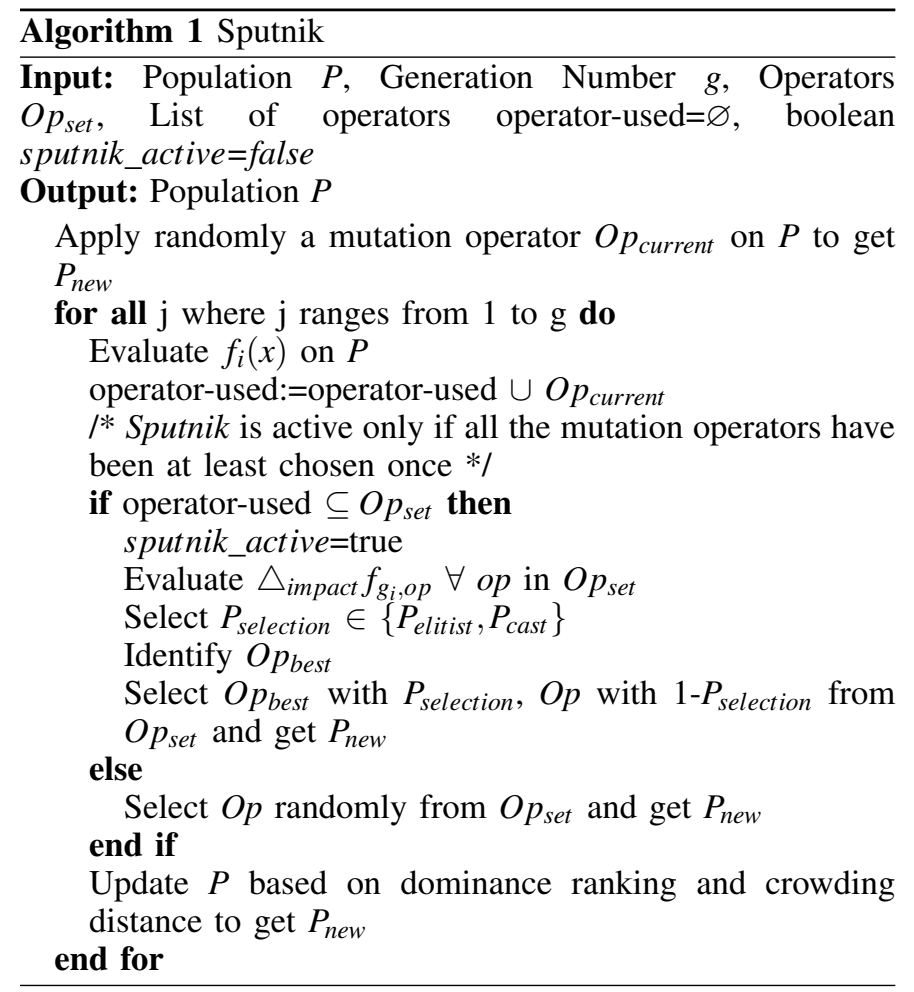

flip in the model that is introduced by an elementary operation. A Cloud infrastructure multi-objective optimization problem is represented by the following Triplet $(I, F, C O)$. I denotes a cloud infrastructure model which represents an abstraction of a set of (VM). Each (VM) hosts $n$ software components (C). $C O$ denotes a set of possible configurations in $I$ that satisfy F. A configuration $c o \in C O$ is obtained through a mapping from Components (C) to Virtual Machines (VMs), for example $c o=\left(V M_{1}\left(c_{1}, c_{2}, c_{3}\right)\right)$ denotes a configuration with a single virtual machine $V M_{1}$ hosting 3 components $c_{1}, c_{2}, c_{3}$. The vector $F(\mathrm{X})$ is composed of the following 4 objective functions, $F(X)=\left(f_{1}(x), f_{2}(x), f_{3}(x), f_{4}(x)\right)$ that have to be minimized. These objectives reflect the different axis of optimization that the provider aims at achieving when placing the software components at the level of the different Virtual Machines and are defined as follows:

- $f_{1}(x)=\operatorname{Cost}(\mathrm{x})$ : Denotes Virtual Machines cost which is proportional to the number of active VMs on the top of a PaaS.

- $f_{2}(x)=$ Isolation( $(\mathrm{x})$ : This function is incremented by 1 whenever two components from different cloud customers share the same Virtual Machine. To achieve isolation, a cloud provider aims at hosting software components belonging to different customers workloads in different Virtual Machines.

- $f_{3}(x)=\operatorname{Similarity}(\mathrm{x})$ : The similarity function quantifies the similarity between the components hosted in Virtual Machines to assess software diversity. Software diversity [2] is an indicator of potential cascading failure to quantify cloud fault tolerance capabilities.
- $f_{4}(x)=\operatorname{Redundancy}(\mathrm{x})$ : The redundancy function provides a score based on redundant software (i.e. number of replicates of the same service).

All fitness values have been normalized to range in the interval $[0,1]$. Table I presents our set of operators $O$ including 7 mutation operators and 1 crossover (SwitchOperator) operator. Software deployment in the cloud is a multi-objective optimization problem that aims at finding a cloud configuration $c o \in C O$ such as min $F(X)$. We have implemented an optimization prototype in the Polymer framework which leverages a model based encoding to perform MOEAs optimization. Model@run.time paradigm [4] enables our cloud models to be seamlessly deployed in a real large-scale production environment [16], [17] like a cloud infrastructure. Thus this validation section aims at evaluating the performance improvement achieved by Sputnik hyper-heuristic, in terms of efficiency and effectiveness to solve the software deployment optimization problem.

\section{A. Research Questions}

This validation section aims at exploring Sputnik efficiency to improve MOEAs convergence speed to achieve a certain level of trade-off between several objectives. Thus, we compare the efficiency achieved with and without Sputnik hyperheuristic. We also explore the effectiveness of Sputnik once embedded in most popular MOEAs algorithms such as $\varepsilon$ MOEA and NSGA-II. As a metric to compare the solutions of the different algorithms under study, we choose the hypervolume [43] metric as Pareto-front quality indicator that defines the total size of space dominated by the solutions in the Pareto-front. Our validation steps are summarized in the following research questions:

- $R Q_{1}$ : Sputnik Efficiency: 1) Considering that an acceptable trade-off is $90 \%$ of the best solutions, is the Darwin Sputnik operator selection strategy successful to reduce the number of generations to reach the defined acceptable trade-off compared to a classical random strategy? 2) What is the gain in terms of execution time of Sputnik compared to MOEAs that are configured without Sputnik? 3) How does Sputnik perform with different probability selection values? 4) What is Sputnik impact on the different objectives functions that have been chosen?

- $R Q_{2}$ : Sputnik Effectiveness: Does Sputnik produce comparable results in terms of trade-offs achieved compared to classical random mutation selection even with modifying the equity of operators selection?

- $R Q_{3}$ : Generalization: What are the applicability limits of Sputnik? How does Sputnik behave with different objectives? How does it behave with the different variants of MOEAs?

\section{B. Experimental results}

To answer $R Q_{1}$, we embed Sputnik in a cloud optimization engine that manages 100 virtual nodes and maintains a web front-end and a load-balancer software components that have 
TABLE I: Operators Definition

\begin{tabular}{|l|l|}
\hline \multicolumn{1}{|c|}{ Operators } & \multicolumn{1}{c|}{ Description } \\
\hline AddVMMutator $\left(\mathrm{VM}_{i}\right.$, PaaS $)$ & Creates a Virtual node $V M_{i}$ on the top of a PaaS \\
\hline AddSoftwareMutator $\left(\right.$ S, $V M_{i}$, PaaS $)$ & Creates a component $S$ in the Virtual node $V M_{i}$ \\
\hline CloneNodeMutator $\left(V M_{i}\right.$, PaaS $)$ & Creates a clone of $V M_{i}$ on the top of PaaS \\
\hline RemoveNodeMutator $\left(\right.$ VM $M_{i}$, PaaS $)$ & Removes a Virtual node $V M_{i}$ on the top of a PaS \\
\hline RemoveSoftwareMutator $(S, P a a S)$ & Removes a software component $S$ from the PaaS \\
\hline AddSmartMutator $(S, P a a S)$ & $\begin{array}{l}\text { Adds a software component } S \text { from the Virtual Node } \\
\text { that contains the least number of components }\end{array}$ \\
\hline RemoveSmartMutator $(S, P a a S)$ & $\begin{array}{l}\text { Removes a component } S \text { from the Virtual Node that } \\
\text { contains the largest number of components }\end{array}$ \\
\hline SwitchOperator $\left(S_{1}, V M_{1}, S_{2}, V M_{2}, P a a S\right)$ & $\begin{array}{l}\text { Switches the component } S_{1} \text { from the Virtual node } V M_{1} \\
\text { to } V M_{2} \text { and switches } S_{2} \text { from the } V M_{2} \text { to } V M_{1}\end{array}$ \\
\hline
\end{tabular}

Fig. 2: Hypervolume: Sputnik (Elitist \& Caste) versus Random Selection

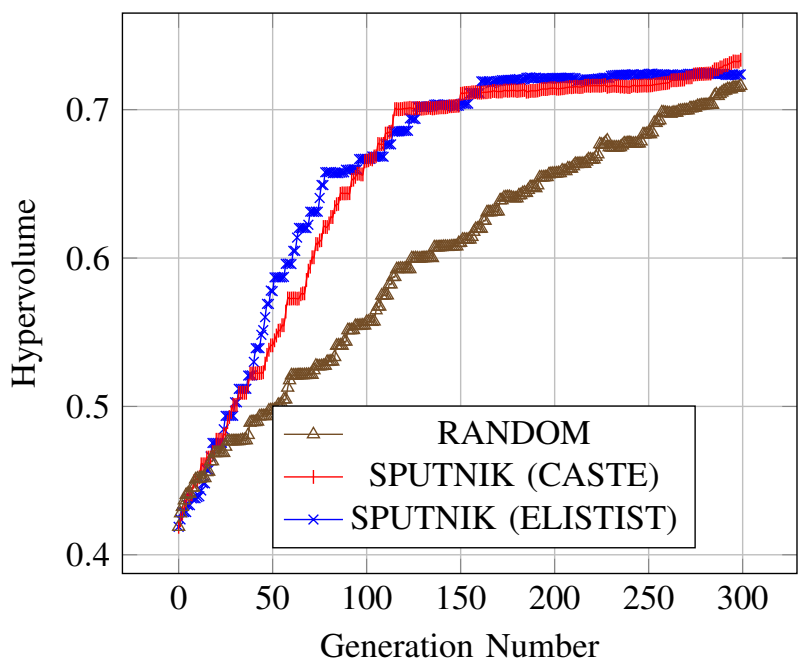

to be dispatched in the different Virtual Machines (VMs). Our cloud reasoning engine is configured to leverage an $\varepsilon$-NSGA II [12]. We perform 30 runs of our experiments with 300 generations with the following configurations: Sputnik with Caste Strategy, Sputnik with Elitist Strategy and finally with random operators selection. The average hypervolume values of the results obtained in the 30 runs, are depicted in Figure 2 according to generation number and in Figure 4.a according to the elapsed time.

We consider that a solution achieves an acceptable trade-off if it reaches $90 \%$ of the best obtained solution. In our case study, the best obtained solution achieves an hypervolume of 0.79 (acceptable hypervolume value is 0.71 in our case), it has been reached with a 250 generations previous run. A run with Sputnik (with both strategies) reaches this value after 176 generations whereas a run with random operators selector reaches this value after 279 generations, respectively 8s for Sputnik and $16 \mathrm{~s}$ for the random selection. Sputnik strategies are very similar in terms of hypervolume achievements, they both reach a value around 0.76 . We notice that elitist strategy converges slightly faster however, the caste strategy can reach better hyper-volume scores. These results can be justified by mutators diversity introduced by the caste strategy, which favors at a certain extent operators equity. In average, Sputnik (both with caste and elitist configurations) outperforms random selection by reducing around $37 \%$ the number of necessary generations, and around $50 \%$ the time to reach acceptable solutions. This confirms our first hypothesis which states that a smart mutation selection strategy is successful to improve efficiency to reach acceptable trade-offs compared to a classical random selection strategy.

To explore the impact of the selection probability of the elitist strategy on Sputnik efficiency, we run the same previous experiment with two different probability values of $P_{\text {selection }}$. The results of a selection probability of $90 \%$ and $50 \%$ are shown in Figure 4.a and Figure 4.b. Unsurprisingly, we observe that the more Sputnik uses its learning strategy, the best is the convergence speed comparing to a random selection.

We also have evaluated the values reached by the different objectives of our case study (Cost, Redundancy, Similarity, Isolation). The results are illustrated in Figure 3. The chart presents the cost per hour and SLA satisfaction percentage reached for the Redundancy, Similarity, Isolation objectives. Note that in a mono-objective optimization that aims at just reducing one objective at a time, we have best objectives values. Note that the values obtained for the mono-objective optimization correspond to distinct runs in which we aimed at optimizing one objective at once on the detriment of other objectives. For our minimization multi-objective optimization problem, Sputnik achieves 3 better objectives values in 400 generations compared to a standard NSGA-II: For the different objectives (Cost, Similarity, Redundancy, Isolation), a possible solution presents the following values $(75.96,29.41$, $78.94,35.57)$ compared to $(76.41,41.26,48.38,57.54)$ for standard NSGA-II. We conclude that for the same number of generations, we obtain results that exhibit better trade-offs with Sputnik activated on top of NSGA-II.

To answer $R Q_{2}$, we run a similar experiment with both 
Fig. 4: NSGA-II Hypervolume with different probabilities of Sputnik Selector

(a) Sputnik selector with a probability of $90 \%$

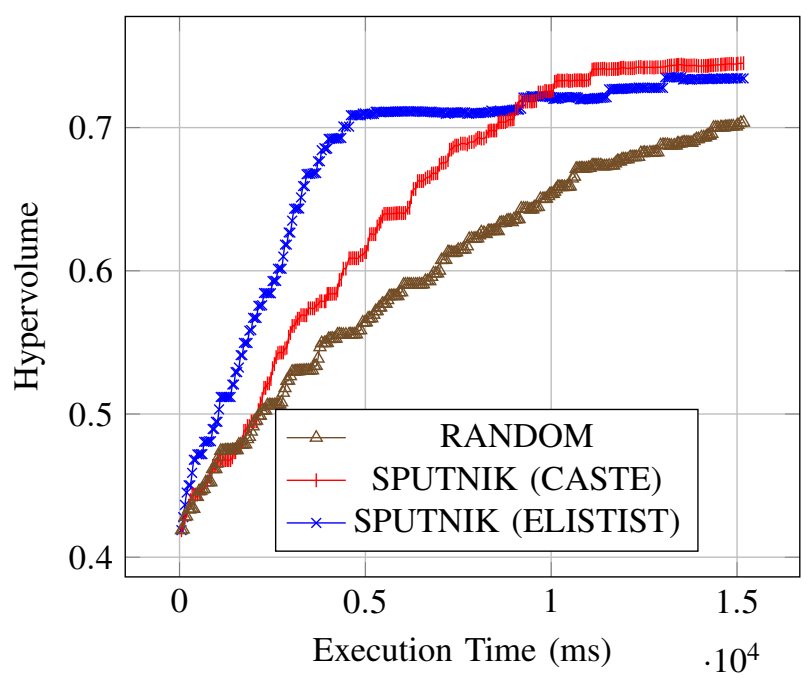

Fig. 3: Objectives Chart Radar

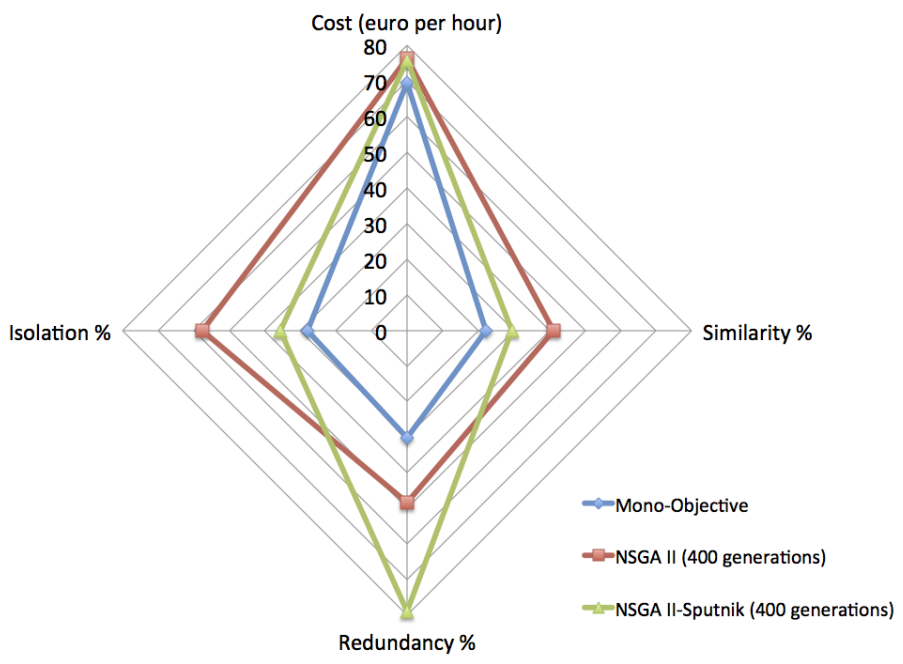

random and Sputnik selector until we reach an unchanged value of hypervolume over 50 generations. Final values for Sputnik (elitist: 0.77 , caste: 0.81 and random 0.78 allow us to conclude that our hyper-heuristic does not decrease the quality of the results in terms of degree of trade-off achieved. Moreover the caste strategy improves the hypervolume score.

To answer $R Q_{3}$, we have evaluated Sputnik with different MOEAs algorithms and various number of objectives. Given that several factors (e.i., implementation aspects, existence of other processes running in the machine, etc) may influence execution time of our approach, we have compared the hypervolume reached over 400 generations. The results are shown (b) Sputnik selector with a probability of $50 \%$

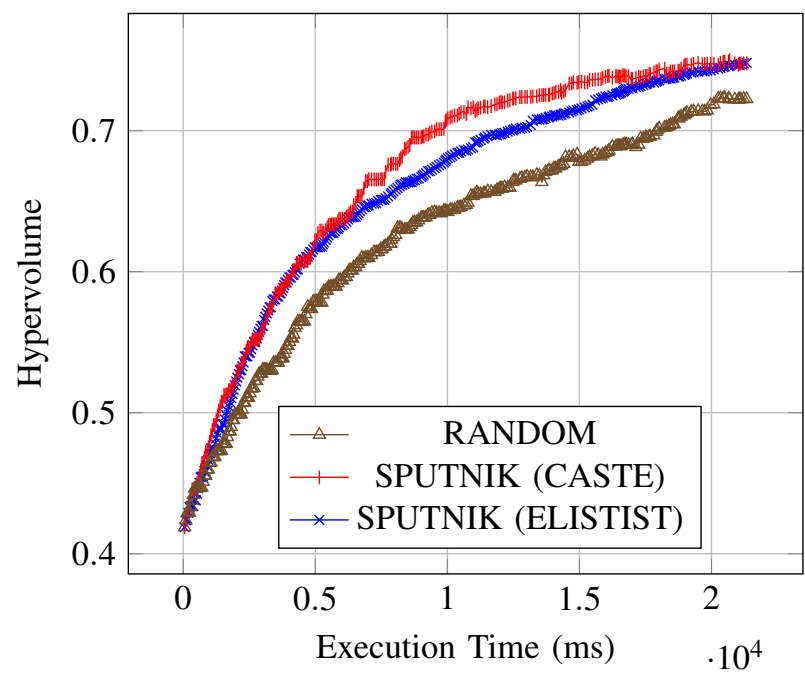

in Figure 5 with hypervolume distribution in Figure 6. For NSGA-II algorithm, an hypervolume value of 0.7 is reached after 90 generations with Elitist strategy, 120 generations with Caste strategy, and reached after 240 generations with random strategy. A Sputnik on top of $\varepsilon$-NSGA-II for almost both the Caste and the Elistist version achieves an hypervolume of 0.6 in 100 generations. Similarly to NSGA-II based approaches, we observe a similar speedup for SMS-MOEA and $\varepsilon$-MOEA algorithms. Above 300 generations for NSGA-II and $\varepsilon$-NSGAII, we also observe that the elitist strategy could lead to little decreased effectiveness. This result could be explained by the total order between operators introduced by elitist strategy, which reduces diversity. We conclude that the caste strategy is less intrusive hyper-heuristic which maintains better the algorithm effectiveness. From these runs, we notice that the Sputnik hyper-heuristic can be generalized on several MOEAs.

Secondly, we have explored the generalization of Sputnik with variable objectives by analyzing the hypervolume while varying the objectives from 1 to 4 . The results, generated with NSGA-II for 200 generations are presented in Figure 7 in term of optimization time. We observe that Sputnik with the caste and elitist strategies provides faster hypervolume convergence compared to random with smaller number of objectives. Indeed for 1 and 3 objectives, the Sputnik strategy selects efficient operators faster, however above 4 objectives, Sputnik effect tends to be similar to random selection. We explain such effect by the fact that Sputnik does not keep the history of previous selected operator selection, thus above 4 objectives, the selection tends to be random. In future work we plan to add operators selection history to maintain Sputnik efficiency independently of the objectives number. 
Fig. 5: Hypervolume over 400 generations

(a) NSGA-II

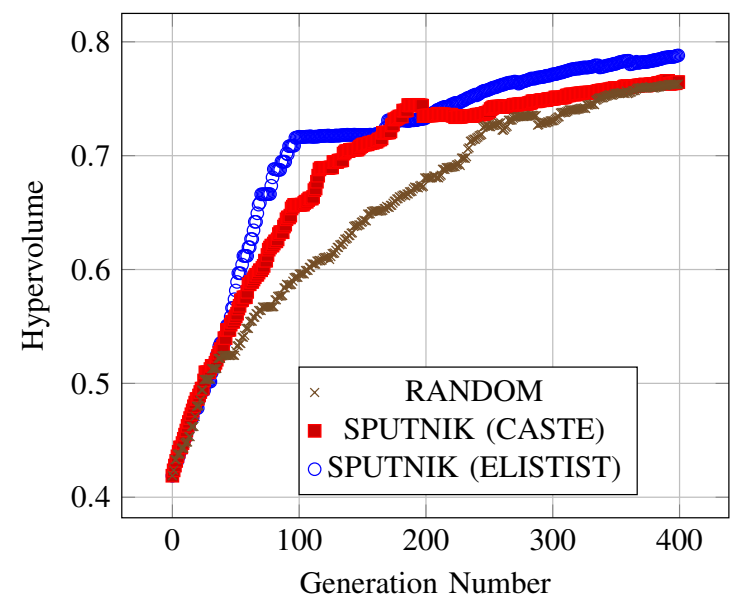

(c) $\varepsilon$-MOEA

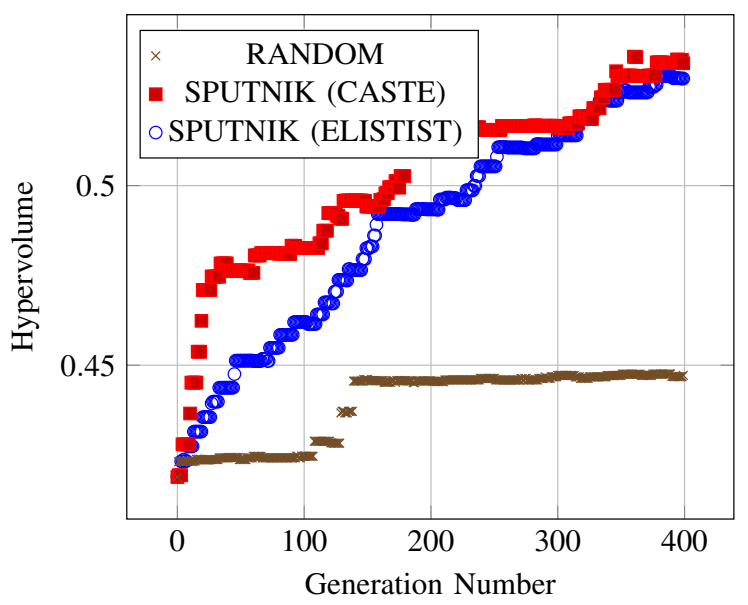

(b) $\varepsilon$-NSGAII

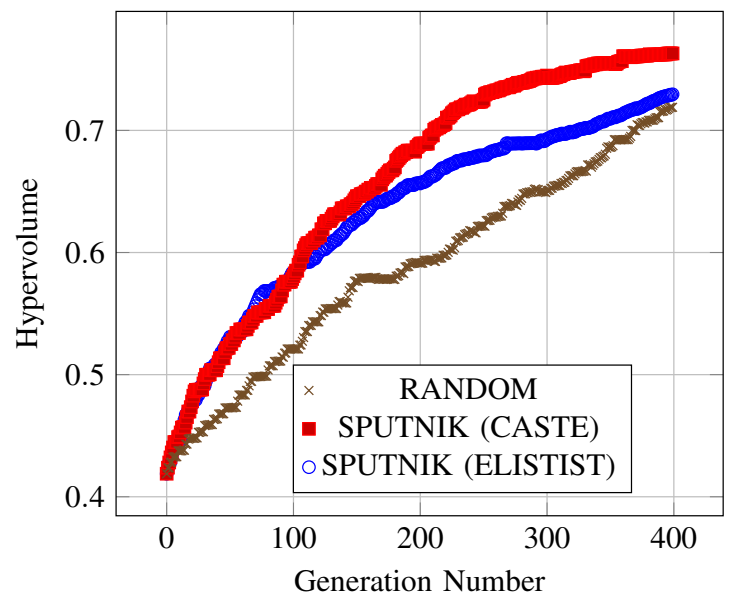

(d) SMS-EMOA

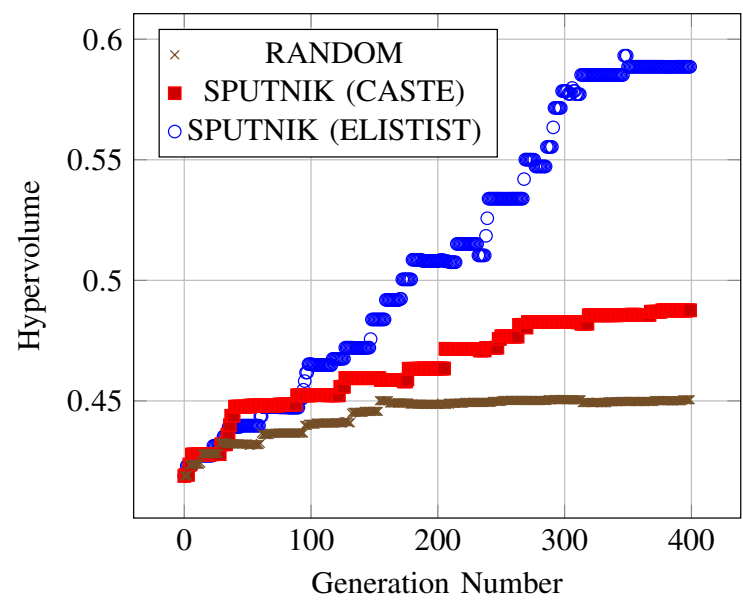

Fig. 6: NSGA-II, $\varepsilon$-NSGAII, $\varepsilon$-MOEA, SMS-EMOA Box Plots

Statistical Distribution for the hypervolume over 400 generations shown above: Max values reached with Sputnik with its two settings outperform max values reached with plain NSGA-II and $\varepsilon$-NSGA-II. $\varepsilon$-MOEA, SMS-EMOA have weaker results due to their selection strategy (one mutation per generation) that slows down Sputnik.

(a) Random

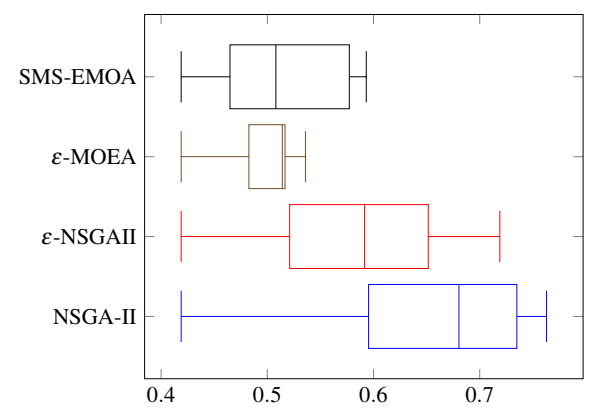

(b) SPUTNIK (ELITIST)

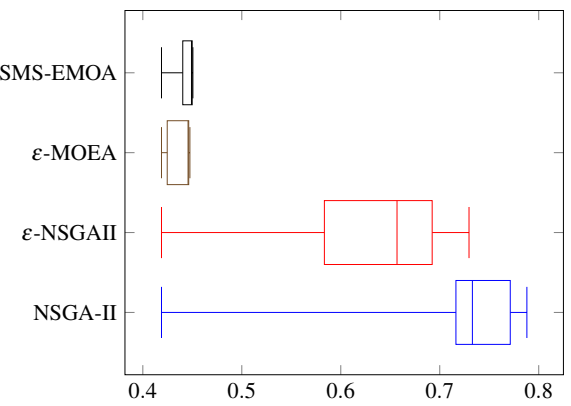

(c) SPUTNIK (CASTE)

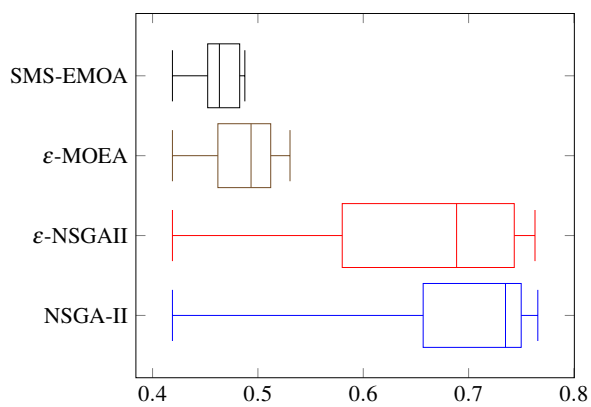


Fig. 7: NSGA-II hypervolume over Execution Time with Variable Objectives

(a) 1 objective

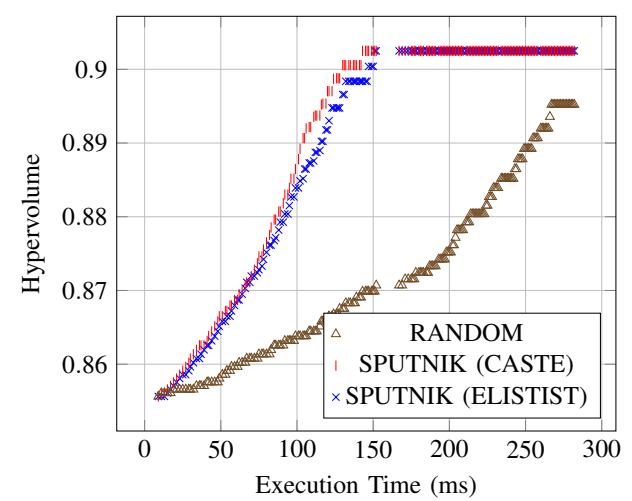

(b) 3 objectives

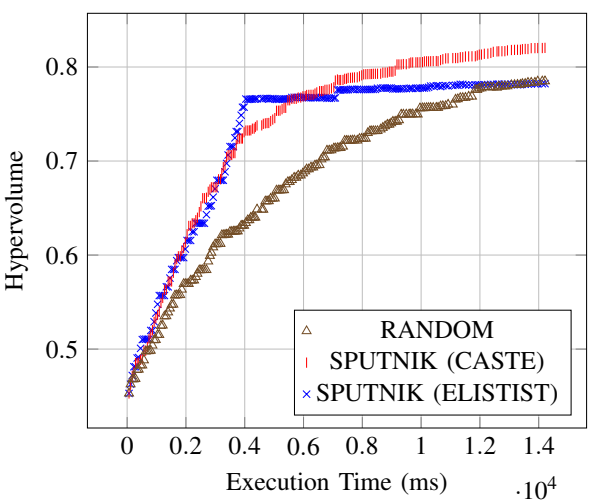

(c) 4 objectives

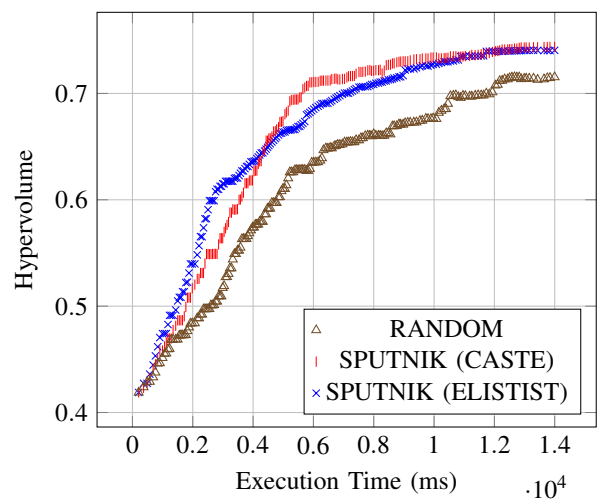

\section{Threats to Validity}

Internal validity is related to the parameters setting of our experiments (i.e. generation number, objectives number, operators, etc...), such as values chosen for the value of $\varepsilon$ in the $\varepsilon$-dominance which might impact our validation results. More specifically, Sputnik incrementally builds a mapping of each operator impact on a particular fitness function. Thus, the coupling effect between the eight used operators and a particular fitness function could introduce a bias on our results. To minimize this bias, we leverage a set of operators without direct coupling effect.

Construct validity arises from the bias introduced in the way we build our experiments. In each experiment presented in the paper, we have compared one run of Sputnik against a random mutator selector. As for any random based techniques, a set of repeated experiments should be run to draw statistially significant results. This bias is mitigated by the number of different experiments that have been run on Sputnik which all demonstrate the effectiveness of the approach.

External validity is related to the generalization of observed results outside the case study presented in the validation section. Although we applied the approach to a cloud reasoning engine and have shown the impact of Sputnik to improve the efficiency of the optimization, further experimentation is needed on different systems that have to comply to run-time constraints in their optimization process.

\section{RELATED WORK}

Several approaches have focused on heuristics to improve classical evolutionary algorithms such as Ant Colony Optimization, Particle Swarm Optimization and Artificial Bee Colony [37]. This work focuses on hyper-heuristics that operate on top of MOEAs to improve their usage in a run-time context and particularly to a cloud-based deployment software. In [36], the authors embed learning techniques in classical MOEAs. They assume that objective functions are expensive to compute so they rank the Pareto front elements and they evaluate only the individuals that have higher ranks. In [30], the authors have proposed an hyper-heuristic that relies on the hypervolume calculation to improve computational results. These approaches consider only the Pareto front set evaluation to improve MOEAs, whereas our approach evaluates operators contribution in improving fitness and thus injects mutation operators that are eligible to make MOEAs converge faster. In [41], the authors have shown that racing algorithms can be used to reduce the computational resources inherent from using evolutionary algorithms in large scale experimental studies, their approach automates solutions selection and discards solutions that do not introduce results improvement. Whereas racing techniques eliminate worst solutions candidates to speed up the search, in our approach we keep considering worst ranked candidates to maintain operators diversity. In [14], the authors have explored the advantages of using a controlled crossover on top of single-point search based hyper-heuristics. They maintain the best solutions obtained during the search and update crossover operator accordingly. The authors rely on a process focused on crossover as a biological selective breeding. This breeding assumes that fittest genes are already present in the initial population. Unlike cited approaches, Sputnik focuses on artificial mutation selection, therefore mimicking the process used to produce genetically modified organisms. As far as we know, there is no other hyper-heuristic that proposes artificial mutation at mutation operators level.

\section{CONCLUSION}

In this paper, we have introduced, an hyper-heuristic breaking the random natural selection of classical MOEAs to leverage an elitist artificial mutation inspired by biological studies [34]. Sputnik relies on a mutation operator selection based on a continuous learning of past effect on fitness functions, instead of random mutation operators selection. The overall goal of Sputnik is to enhance the optimization algorithm itself, and to guide the search towards faster trade-offs achievement to finally save generation cycles and time. Experimentally, we provide evidence of the effectiveness of artificial mutation to reduce significantly the number of necessary generations to find acceptable trade-offs. In the future, we plan to explore the effectiveness of Sputnik in accelerating the optimization 
processes of other adaptive systems that have to comply with run-time constraints.

\section{REFERENCES}

[1] Deriving operating policies for multi-objective reservoir systems: Application of self-learning genetic algorithm. Applied Soft Computing, 10(4):1151 - 1163, 2010.

[2] B. Baudry, M. Monperrus, C. Mony, F. Chauvel, F. Fleurey, S. Clarke, et al. Diversify-ecology-inspired software evolution for diversity emergence. In CSMR.

[3] D. Beasley, R. Martin, and D. Bull. An overview of genetic algorithms: Part 1. fundamentals. University computing, 15:58-58, 1993.

[4] G. Blair, N. Bencomo, and R. B. France. Models@ run. time. Computer, 42(10):22-27, 2009.

[5] D. Buche, N. N. Schraudolph, and P. Koumoutsakos. Accelerating evolutionary algorithms with gaussian process fitness function models. Systems, Man, and Cybernetics, Part C: Applications and Reviews, IEEE Transactions on, 35(2):183-194.

[6] E. K. Burke, M. Hyde, G. Kendall, G. Ochoa, E. Özcan, and J. R Woodward. A classification of hyper-heuristic approaches. In Handbook of Metaheuristics, pages 449-468. Springer, 2010.

[7] S. Chaisiri, B.-S. Lee, and D. Niyato. Optimization of resource provisioning cost in cloud computing. Services Computing, IEEE Transactions on, 5(2):164-177, 2012.

[8] C.-Y. Chao. Using security levels to improve permission checking performance and manageability, 2009.

[9] Q. Chen, P. Grosso, K. van der Veldt, C. de Laat, R. Hofman, and $\mathrm{H}$. Bal. Profiling energy consumption of vms for green cloud computing. In Dependable, Autonomic and Secure Computing (DASC), 2011 IEEE Ninth International Conference on, pages 768-775, 2011.

[10] C. A. C. Coello and G. B. Lamont. Applications of multi-objective evolutionary algorithms, volume 1. 2004.

[11] C. Darwin. On the origins of species by means of natural selection. 1859

[12] K. Deb et al. Multi-objective optimization using evolutionary algorithms, volume 2012. John Wiley \& Sons Chichester, 2001.

[13] K. Deb, L. Thiele, M. Laumanns, and E. Zitzler. Scalable multiobjective optimization test problems. In Proceedings of the Congress on Evolutionary Computation (CEC-2002),(Honolulu, USA), pages 825830, 2002.

[14] J. H. Drake, E. Özcan, and E. K. Burke. Controlling crossover in a selection hyper-heuristic framework. School of Computer Science, University of Nottingham, Tech. Rep. No. NOTTCS-TR-SUB-11041816384244, 2011.

[15] D. El Kateb, F. Fouquet, G. Nain, J. A. Meira, M. Ackerman, and Y. Le Traon. Generic cloud platform multi-objective optimization leveraging models@ run. time. 2014.

[16] F. F. et al. Dissemination of reconfiguration policies on mesh networks. In DAIS, 2012.

[17] F. Fouquet, B. Morin, F. Fleurey, O. Barais, N. Plouzeau, and J.-M. Jezequel. A dynamic component model for cyber physical systems. In Proceedings of the 15th ACM SIGSOFT symposium on Component Based Software Engineering, pages 135-144. ACM, 2012.

[18] S. Frey, F. Fittkau, and W. Hasselbring. Search-based genetic optimization for deployment and reconfiguration of software in the cloud. In Proceedings of the 2013 International Conference on Software Engineering, pages 512-521, 2013.

[19] M. Friedman. A Theory of the Consumption. 1957.

[20] I. Güney, G. Küçük, and E. Özcan. Hyper-heuristics for performance optimization of simultaneous multithreaded processors. In Information Sciences and Systems 2013, pages 97-106, 2013.

[21] M. Harman, K. Lakhotia, J. Singer, D. R. White, and S. Yoo. Cloud engineering is search based software engineering too. Journal of Systems and Software, 86(9):2225-2241, 2013.
[22] E. B. i Mansilla and J. M. G. i Guiu. Molecs: Using multiobjective evolutionary algorithms for learning. In Evolutionary Multi-Criterion Optimization, pages 696-710, 2001

[23] H. Ishibuchi, Y. Nojima, and T. Doi. Comparison between singleobjective and multi-objective genetic algorithms: Performance comparison and performance measures. In Evolutionary Computation, 2006. CEC 2006. IEEE Congress on, 2006.

[24] H. Ishibuchi, Y. Sakane, N. Tsukamoto, and Y. Nojima. Evolutionary many-objective optimization by nsga-ii and moea/d with large populations. In Systems, Man and Cybernetics, 2009. SMC 2009. IEEE International Conference on, pages 1758-1763, Oct 2009.

[25] M. T. Jensen. Reducing the run-time complexity of multiobjective eas: The nsga-ii and other algorithms. Evolutionary Computation, IEEE Transactions on, 7(5):503-515, 2003.

[26] Y. Jin. A comprehensive survey of fitness approximation in evolutionary computation. Soft computing, 9(1):3-12, 2005.

[27] G. Jung, M. A. Hiltunen, K. R. Joshi, R. D. Schlichting, and C. Pu. Mistral: Dynamically managing power, performance, and adaptation cost in cloud infrastructures. In Distributed Computing Systems (ICDCS), 2010 IEEE 30th International Conference on, pages 62-73, 2010.

[28] J. O. Kephart and D. M. Chess. The vision of autonomic computing Computer, 36(1):41-50, 2003.

[29] V. Khare, X. Yao, and K. Deb. Performance scaling of multi-objective evolutionary algorithms. In Proceedings of the 2Nd International Conference on Evolutionary Multi-criterion Optimization, EMO'03, pages 376-390, 2003.

[30] C. León, G. Miranda, and C. Segura. Hyperheuristics for a dynamicmapped multi-objective island-based model. In Distributed Computing, Artificial Intelligence, Bioinformatics, Soft Computing, and Ambient Assisted Living, pages 41-49. 2009.

[31] S. W. Mahfoud. Niching methods for genetic algorithms. Urbana, 51(95001), 1995.

[32] E. Özcan, B. Bilgin, and E. E. Korkmaz. A comprehensive analysis of hyper-heuristics. Intelligent Data Analysis, 12(1):3-23, 2008.

[33] P. Patel, A. H. Ranabahu, and A. P. Sheth. Service level agreement in cloud computing. 2009.

[34] H. PLASKETT. Artificial transmutationof the gene. tic, 66(1699), 1927.

[35] C. R. Reeves and J. E. Rowe. Genetic algorithms: principles and perspectives: a guide to GA theory, volume 20. 2003.

[36] C.-W. Seah, Y.-S. Ong, I. W. Tsang, and S. Jiang. Pareto rank learning in multi-objective evolutionary algorithms. In Evolutionary Computation (CEC), 2012 IEEE Congress on, pages 1-8, 2012.

[37] V. Selvi and D. R. Umarani. Comparative analysis of ant colony and particle swarm optimization techniques. International Journal of Computer Applications Volume, 2010.

[38] K. C. Tan, T. H. Lee, and E. F. Khor. Evolutionary algorithms with dynamic population size and local exploration for multiobjective optimization. Evolutionary Computation, IEEE Transactions on, 5(6):565588, 2001.

[39] D. A. Van Veldhuizen. Multiobjective evolutionary algorithms: classifications, analyses, and new innovations. $\mathrm{PhD}$ thesis, 1999.

[40] D. A. Van Veldhuizen and G. B. Lamont. Multiobjective evolutionary algorithms: Analyzing the state-of-the-art. Evolutionary computation, 8(2):125-147, 2000

[41] B. Yuan and M. Gallagher. Statistical racing techniques for improved empirical evaluation of evolutionary algorithms. In Parallel Problem Solving from Nature-PPSN VIII, pages 172-181, 2004.

[42] A. Zhou, B.-Y. Qu, H. Li, S.-Z. Zhao, P. N. Suganthan, and Q. Zhang. Multiobjective evolutionary algorithms: A survey of the state of the art. Swarm and Evolutionary Computation, 1(1):32-49, 2011.

[43] E. Zitzler, D. Brockhoff, and L. Thiele. The hypervolume indicator revisited: On the design of pareto-compliant indicators via weighted integration. In Evolutionary Multi-Criterion Optimization, pages 862876, 2007. 